\title{
Self Shadow Elimination Algorithm for Surveillance Videos using Inferential Difference in Mean Statistics
}

\author{
Girisha R \\ PET Research Center \\ P.E.S. College of Engineering \\ Mandya, Karnataka, India
}

\author{
Murali S \\ PET Research Center \\ P.E.S. College of Engineering \\ Mandya, Karnataka, India
}

\begin{abstract}
Identifying moving objects from a video sequence is a fundamental and critical task in many computer vision applications and a robust segmentation of motion objects from the static background is generally required. Segmented foreground objects generally include their self shadows as foreground objects since the shadow intensity differs and gradually changes from the background in a video sequence. Moreover, self shadows are vague in nature and have no clear boundaries. To eliminate such shadows from motion segmented video sequences, we propose an algorithm based on inferential statistical Difference in Mean (Z) method. This statistical model can deal scenes with complex and time varying illuminations without restrictions on the number of light sources and surface orientations. Results obtained with different indoor and outdoor sequences show that algorithm can effectively and robustly detects associated self shadows from segmented frames.
\end{abstract}

\section{Keywords}

Video Surveillance, Motion Segmentation, Self Shadows, Inferential Statistics, Difference in Mean Method.

\section{INTRODUCTION}

Analysis of human movement is currently one of the most active research topics in computer vision. Human Motion Analysis (HMA) includes detection, tracking and recognition of people. HMA can be classified into 3 categories [1, 2], namely low level vision (Detection), intermediate level vision (Tracking) and high level vision (Behavioral Analysis). The application domains where HMA can be applied are video surveillance, content-based image retrieval, gait recognition etc.

The automated video surveillance system was expected to detect people, monitor their actions, and subsequently need to analyze their behavior in order to prevent any untoward incidents. To analyze the behavior of a person in a given setup, the first step is human detection and tracking. Tracking involves detection of regions of interest in a frame and then finding frame-to-frame correspondence of each region's location and shape.

Nearly, every system in the HMA starts with segmentation $[1,2,3]$; current motion segmentation methods are mainly based on background subtraction or temporal differencing or optical flow or adaptive background model $[1,2]$. One of the main challenges after segmentation is identifying self shadows $[4,6]$. Segmented foreground objects generally includes their self shadows as a foreground object since the shadow intensity differs from the background. To obtain a better segmentation quality, object tracking algorithms must correctly separate foreground objects from the shadows. Since, self shadows produce troublesome effects for video surveillance systems, typically for motion tracking from a fixed viewpoint because sometimes they may appear as segmented object as shown in Fig. 2. Despite many attempts, the problem remains largely unsolved, due to several inherent challenges: Dark regions are not necessarily self shadow regions since foreground objects can be dark too; A commonly used assumption is that these shadows falls only on the segmented object is not valid to general scenes as shown in Fig. 2.

In this paper, we propose a self shadow elimination method based on inferential statistics using Difference in Mean $(Z)$ method, which does not put any restrictions on the scene in terms of illumination conditions, geometry of the objects and size and position of shadows. Results obtained using the proposed approach, in varied conditions, are very promising. The rest of this paper is organized as follows: Section 2 presents a review of the recent and ongoing activity in the domain of self shadow elimination and then briefly explains outline of the approach; section 3 discusses proposed methodology to identify self shadow pixels and elimination of them subsequently. Section 4 discusses experimental results; finally, section 5 concludes the proposed methodology with summary.

\section{RELATED WORK}

It is very common in real world that the shadow will appear as long as an object is in front of the light source. It is not difficult for human eyes to distinguish shadows from objects. However, identifying shadows by computer is a challenging research problem. Shadows occur when objects totally or partially occlude direct light from a light source. According to the classification reported [6] shadows are composed of two parts: self shadows and cast shadows. The self shadow is the part of the object, which is not illuminated by the light source as shown in the Fig. 2 . The cast shadow is the area projected on the scene by the object as shown in Fig. 1(e) and is further classified into umbra and penumbra. The umbra corresponds to the area where the direct light is totally blocked by the object, where as in the penumbra area is partially blocked.

Self shadow detection and elimination algorithms can be classified into model or property based techniques. Model based techniques are usually used for specific situations such as in $[5,7$, $8,9,10]$, where priori knowledge of scene geometry and foreground objects is incorporated into a model. Property based approaches [11], uses features like geometry, brightness or color 
to identify shadow regions, are more robust to different scene and illumination conditions. this paper, we only present a simple and novel algorithm to eliminate self shadows from segmented foreground objects.

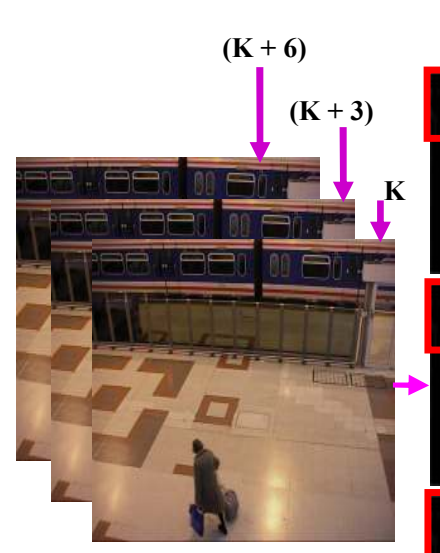

1(a)

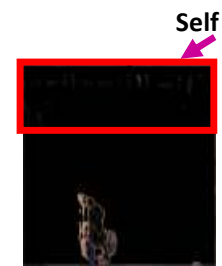

Self Shadows
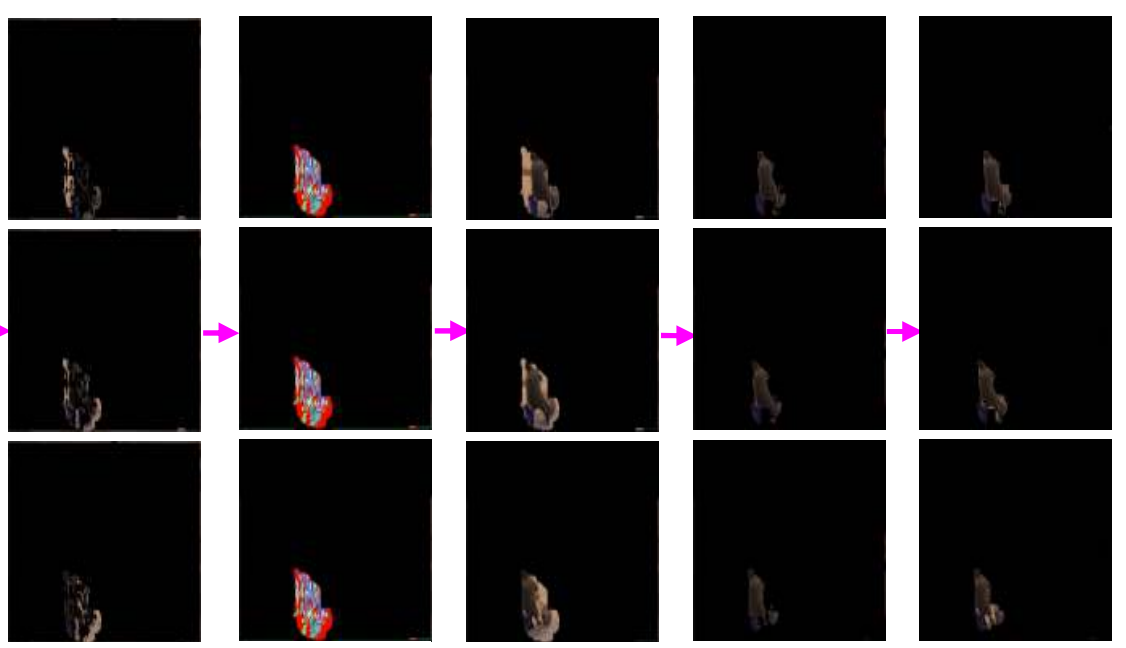

1(f)

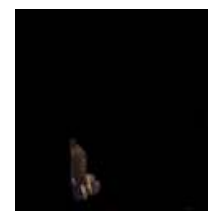

1(e)

1(d)

$1(\mathrm{~g})$

Figure 1. Overview of proposed system to segment motion objects from surveillance video frames.

(a) Input frames 109, 112 and 115 of the PETS 2006 data set 7, camera 3. (b) Motion segmentation. (c) Self shadow elimination. (d) Spatial Clustering. (e) Segmented Object. (f) Cast shadow elimination. (g) Segmented foreground object

The method proposed in [7] uses photometric color invariants to extract shadow regions and subsequently classified as self (if on the object) or cast (if on the ground plane) shadows. Where, in [8] shadowing factor is derived as a function of surface roughness and in color variation; and assumes surface is homogeneous, isotropic and smooth microscopically with a Gaussian height field. Shadow light environment is estimated in an image [10], using cast and self shadows in a real image. Both self and cast shadows are eliminated from static images in [11]. First self shadows are eliminated using gradient space and then cast shadow edges are extracted using color invariants finally, using Poisson equation shadow free reflectance image is obtained.

Presences of shadows are determined first using illumination direction in [9]. Object shapes are recovered using object edges if shadows are present. [9] Eliminates the cast shadows from the outdoor images if it is on the ground plane and it keeps the shadows on the object as self shadows based on HSI color space. However, this technique cannot be applied to dynamic environments because method is based on background subtraction; assumes self shadows occur only on the object and nowhere else.

Fig. 1, depicts an overall scheme to segment motion objects from background and to eliminate shadows (Self and Cast) from the segmented motion objects using PETS video of 2006, data set 7 of camera 3 for video frames 109, 112 and 115. We use multiple correlation [3] to segment motion objects from temporal differencing frames. After motion segmentation, we apply $\mathrm{Z}$ to eliminate self shadows as shown in Fig. 1(c) and then spatial clustering is applied as shown in Fig. 1(d) because temporal differencing generates holes in segmented objects [3]. Cast shadows are eliminated using standard scores [15] as shown in Fig. 1(f). Again, spatial clustering applied to group motion objects to get final segmented object as shown in Fig. 1(g). However, in

\subsection{Outline of the Overall Scheme}

Motion segmentation of foreground objects is an active area of research and a number of techniques have been developed over a decade. The proposed self shadow elimination algorithm in this paper, takes an initial set of foreground pixels and then attempts to classify them either as motion objects or as self shadows. For that, we use proposed methodology [3] to segment three frames $(\mathrm{K}$, $(\mathrm{K}+3)$ and $(\mathrm{K}+6))$ simultaneously, which is based on statistical multiple correlation coefficient and temporal differencing. The proposed motion segmentation algorithm [3] robust to illuminations, complex backgrounds, adapts to dynamic environments and reflections can vary without significantly affecting the result.

Motion segmentation is done [3], by checking pixel by pixel disparity in RGB color space between three (by using equation (3)) video frames simultaneously as shown in Fig. 1(b). Image subtraction is based on temporal differencing (frame gap is three) between $\mathrm{K},(\mathrm{K}+3)$ and $(\mathrm{K}+6)$ as shown in Fig. 4. Extensive experiments conducted by us on PETS data set revealed that, if we do temporal difference with successive frames as shown in Fig. 3 (i.e., $\mathrm{K},(\mathrm{K}+1)$ and $(\mathrm{K}+2))$ motion of the objects is almost negligible and its waste of processing time. On the other hand, if we increase frame gap beyond three frames than the objects moved very fast in the scene and generated unnecessary cast shadows in the corresponding difference images as shown in Fig. 5 .

Self shadows are modeled based on $\mathrm{Z}_{\mathrm{ML}}$ after foreground pixel extraction [3]. $Z_{\mathrm{ML}}$ value is computed between frames ( $\mathrm{K}$ and $(\mathrm{K}+3))$ and $((\mathrm{K}+3)$ and $(\mathrm{K}+6))$, among corresponding RGB values of the pixels $\mathrm{P}(\mathrm{x}, \mathrm{y})$ using equation (1). Finally, the average of the two computed $Z$ value is taken from equation (4) to decide if the current sample pixel $\mathrm{P}(\mathrm{x}, \mathrm{y})$ belongs to self shadow or to motion object as represented in equation (2). Those parts of the 
segmented motion objects, which are not illuminated by light source, become self shadows such parts are also eliminated by self shadow removal algorithm.
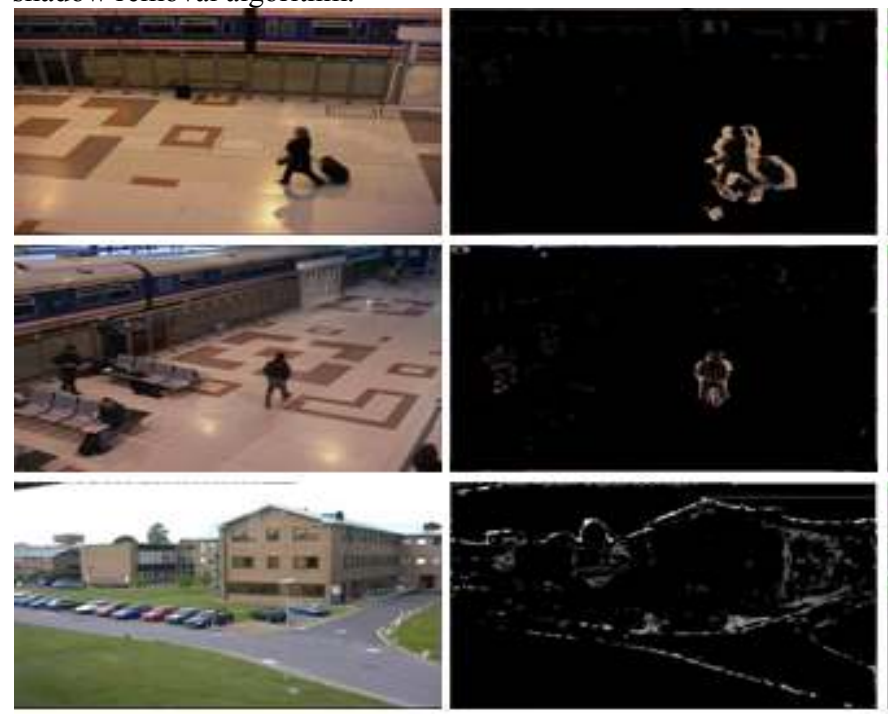

Figure 2. Self shadow identification and elimination from PETS 2006 and 2001 video frames.

First column: Input frame; Second column: Motion segmented output from [3]; Third column: Identified self shadows (Green color) from the proposed algorithm; Fourth column: Self shadows eliminated by the proposed algorithm

The temporal differencing is very adaptive to dynamic environment, but generally does a poor job of extracting all relevant feature pixels i.e., segmented regions contains holes in motion objects. To fill the holes of the blob, we use spatial clustering (the criterion is spatial distance between pixels) first applied horizontally and then vertically as shown in Fig. 1(d). Drawback of this step is that cast shadow area may increase as shown in Fig. 1(e).

The cast shadows removed if any, using standard scores in [15] shown in Fig. 1(f). Cast shadow detection and elimination algorithm [15] first detects possible cast shadow pixels by scanning video frames and represents first encountered motion segmented pixel RGB values as standard scores' parameter. Then again video frame is scanned and encountered motion segmented pixels are represented as standard scores. If the standard scores is less than the predefined critical value then that particular pixel is considered as cast shadow. The proposed algorithm [15] updates its standard scores for every input frame and it not only eliminates penumbra it also eliminates umbra from the segmented object as shown in Fig. 1(f). After cast shadow elimination, again spatial cluster is applied as shown in Fig. 1(g).

\subsection{Outline of the self shadow algorithm}

The two main theoretical branches of statistical science are descriptive and inferential statistics. The former is useful to characterize the overall set of data, called population, by assigning a proper descriptive model or distribution family to it. The latter one, adapted when the entire set of data is unknown and we want to infer the behavior of the entire population from a sub-set of sample data $[12,13,14]$.

We use inferential statistics Difference in Mean $(Z)$ test in this paper, for comparing means of two independent populations. The $\mathrm{Z}$ uses two components null hypothesis $\left(\mathrm{H}_{0}\right)$ and alternative hypothesis $\left(\mathrm{H}_{1}\right)$ to test a claim (i.e., two sample means are equal or not). A hypothesis is a claim or statement about a property of population [12]. Where, the $\mathrm{H}_{0}$ is a claim about a population parameter that is assumed true until it is declared false. Where, $\mathrm{H}_{1}$ is claim about a population parameter that will be true if the $\mathrm{H}_{0}$ is
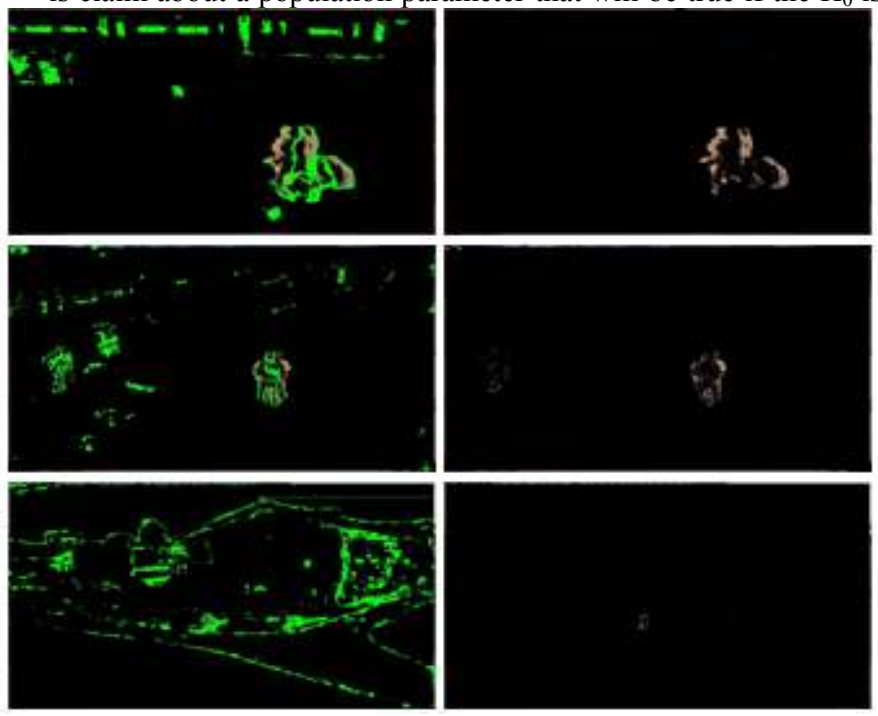
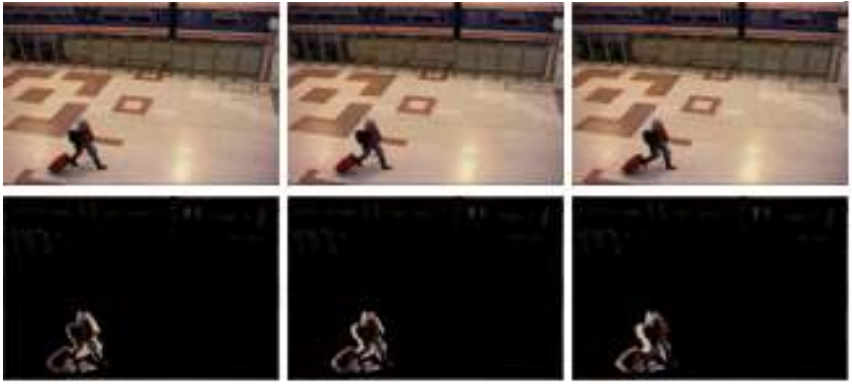

Figure 3. Frames 487, 488 \& 489 of a PETS 2006 data set 5. First row: Input frames. Second row: Output of [3]
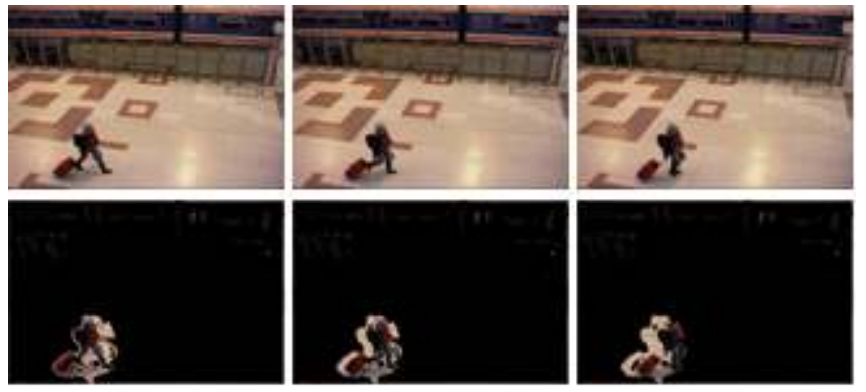

Figure 4. Frames 487, 490 \& 493 of a PETS 2006 data set 5. First row: Input frames. Second row: Output of [3]
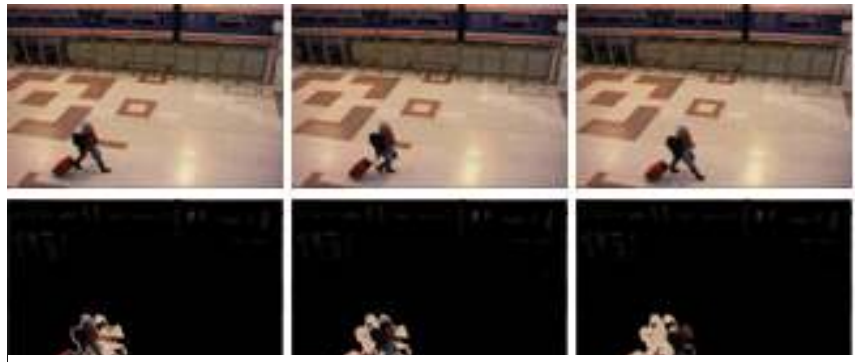

Figure 5. Frames 487, $492 \& 497$ of a PETS 2006 data set 5. First row: Input frames. Second row: Output of [3] 
false. The $\mathrm{Z}$ test in this paper uses a predetermined significance level, denoted by $\alpha$ to test a claim (i.e., probability of rejecting $\mathrm{H}_{0}$ ). A confidence interval is the range of values that we believe to be part of the $\mathrm{H}_{0}$ population (i.e., that would lead us to retain the $\mathrm{H}_{0}$ ) is constructed using critical values in such a way that the probability of rejecting the $\mathrm{H}_{0}$, if it is true, is equal to $\alpha$ [13]. Critical values (are selected from standard normal distribution table depending on $\alpha$ value) separates the critical region (where we reject the $\mathrm{H}_{0}$ ) from the values of the $\mathrm{Z}$ test statistics that do not lead to a rejection of the $\mathrm{H}_{0}$ as shown in Fig. 7.

Let, the pixel RGB value on any coordinate $(x, y)$ is denoted by $\mathrm{p}(\mathrm{x}, \mathrm{y})$ with $\mathrm{x}$ in the range from 0 to $\mathrm{W}_{\mathrm{X}}$ and $\mathrm{y}$ in the range 0 to $\mathrm{h}_{\mathrm{y}}$. Where, $W_{X}$ and $H_{Y}$ are the size of the image in the $X$ and $Y$ directions, respectively. Let, a pixel $\mathrm{p}(\mathrm{x}, \mathrm{y})$ along with its eight neighbors $(\mathrm{N} 8(\mathrm{p}))$ from now on referred to as sample pixel $\mathrm{P}(\mathrm{x}, \mathrm{y})$ as shown in Fig. 6. The idea at the basis of the proposed method is to infer current sample pixel $\mathrm{P}(\mathrm{x}, \mathrm{y})$ in all three temporal differencing frames either it belongs to self shadows (if $\mathrm{H}_{0}$ is true) or it belongs to motion objects (if $\mathrm{H}_{1}$ is true).

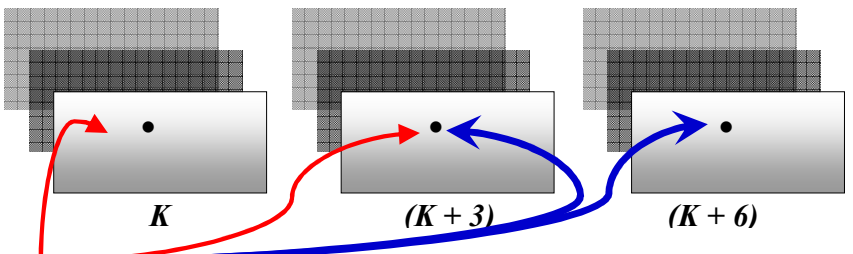

$p(x, y)$ and its eight neighbors $(N 8(p))$ referred as pixel $P(x, y)$ in each frame and at a given time 2 frames $P(x, y)$ RGB (in total 27 from 9 pixels) values are used in each $Z$ calculation.

Figure 6. Pixels selection for $Z$ calculation.

\section{SELF SHADOW ELIMINATION}

In this section, we consider statistical inferences of $\mathrm{Z}$ test, to eliminate self shadows. Let, $\mu_{M}$ be the mean of the first population and $\mu_{\mathrm{L}}$ be the mean of the second population. To test a hypothesis about the difference between these two populations means i.e. $\left(\mu_{\mathrm{M}}\right.$ - $\mu_{\mathrm{L}}$ ), we calculate $\left(\mathrm{X} \square_{\mathrm{M}}-\mathrm{X} \square_{\mathrm{L}}\right.$ ) to make an interval estimate and to test a hypothesis. Where, $X \square_{M}$ be the mean of a sample taken from the first population and $\mathrm{X} \square_{\mathrm{L}}$ be the mean of a sample taken from the second population [12]. Considering following two possibilities $\mathrm{H}_{0}$ and $\mathrm{H}_{1}$, based on independent random samples of size $n_{M}=27$ and $n_{L}=27$ of the two temporal frames as shown in Fig. (6). Therefore, the sampling distribution of $\left(X \square_{M}-X \square_{L}\right)$ is large and approximately normal, and we use normal distribution to perform the hypothesis test [14].

$$
\begin{gathered}
\text { Let, } \mathrm{H}_{0:} \mu_{\mathrm{M}}-\mu_{\mathrm{L}}=0 \text { (Belongs to Self Shadow) } \\
\text { Let, } \mathrm{H}_{1:} \mu_{\mathrm{M}}-\mu_{\mathrm{L}} \neq 0 \text { (Belongs to Motion Object) } \\
Z_{\mathbf{L L}}=\frac{\left(\overline{X_{\mathbf{L}}}-\overline{X_{L}}\right)-\left(\mu_{\mathbf{L}}-\mu_{\mathbf{L}}\right)}{\sqrt{\frac{S_{\mathbf{L}}^{t}}{n_{\mathbf{L}}}+\frac{S_{L}^{\mathbf{L}}}{n_{L}}}}
\end{gathered}
$$

Where, $Z_{M L}$ is the Difference in Means test of the pixel $P(x, y)$ between two temporal differencing frames RGB values. The $Z_{M L}$ is calculated for each $\left(W_{X} \times H_{y}\right)$ remaining foreground pixels of the [3] frames. Where, $\mathrm{M}=\{\mathrm{K},(\mathrm{K}+3)\}, \mathrm{L}=\{(\mathrm{M}+3)\} . \mathrm{n}_{\mathrm{M}}=\mathrm{n}_{\mathrm{L}}=$ 27 are number of RGB values of the pixel $P(x, y)$. Where, the value of $\left(\mu_{M}-\mu_{L}\right)=0$ substituted from $H_{0}$. Where, $S_{M}$ and $S_{N}$ are the standard deviations of the two samples selected from the $\left(\mathrm{X} \square_{\mathrm{M}}-\mathrm{X} \square_{\mathrm{L}}\right)$.

Let $\mathrm{I}^{\mathrm{K}}, \mathrm{I}^{(\mathrm{K}+3)}$ and $\mathrm{I}^{(\mathrm{K}+6)}$ are motion segmented frames of $\mathrm{K}^{\text {th }}$, $(\mathrm{K}+3)^{\text {th }}$ and $(\mathrm{K}+6)^{\mathrm{th}}$ respectively after [3] as shown in Fig. 1(a). Then images $\mathrm{D}^{\mathrm{K}}, \mathrm{D}^{(\mathrm{K}+3)}$ and $\mathrm{D}^{\mathrm{K}+6)}$ are generated using equation (2) which contains self shadow eliminated motion objects of frames $K^{\text {th }},(K+3)^{\text {th }}$ and $(K+6)^{\text {th }}$ respectively as shown in Fig. 1(c).

$$
\begin{gathered}
D_{(x, y)}^{i}=\left\{\begin{array}{l}
0, \text { if }\left(|Z| \leq T_{S}\right) \\
R G B \text { of } I_{(x, y)}^{i} \text { if }\left(|Z|>T_{S}\right)
\end{array}\right. \\
\mathrm{I}=\{\mathrm{K},(\mathrm{K}+3),(\mathrm{K}+6)\} \\
\mathrm{K}=(9 \mathrm{n}+1)^{\text {th }} \text { frame, where } \mathrm{n} \geq 0 \text { and } \mathrm{n} \square \mathrm{N} \\
Z=\frac{Z_{K,(K+3)+} Z_{(K+3),(K+6)}}{2}
\end{gathered}
$$

Where, $\mathrm{Z}$ is an average value taken from $\mathrm{Z}_{\mathrm{K},(\mathrm{K}+3)}$ and $\mathrm{Z}_{(\mathrm{K}+3),(\mathrm{K}+6)}$ (both values are computed using $Z_{M L}$ ) and $T_{S}$ is a critical value empirically chosen from standard normal curve table [14]. The significance level $(\alpha)$ is 0.01 . The $\neq$ sign in the $\mathrm{H}_{1}$ indicates that the test is two-tailed. A two-tailed test has rejection regions in both tails. The area in each tail of normal distribution curve will be $\alpha / 2=0.01 / 2=0.005$. The critical values of the $Z$ for 0.005 areas in each tail of the normal distribution curve are $\approx 2.58$ and 2.58 from standard normal distribution table.

Fig. 7, shows $Z$ confidence interval for PETS 2006, data set 7, camera 3 video sequence. Critical value range $\left(T_{S}= \pm 2.58\right)$ is compared with calculated $\mathrm{Z}$ value at 99 confidence level and if $\mathrm{Z}$

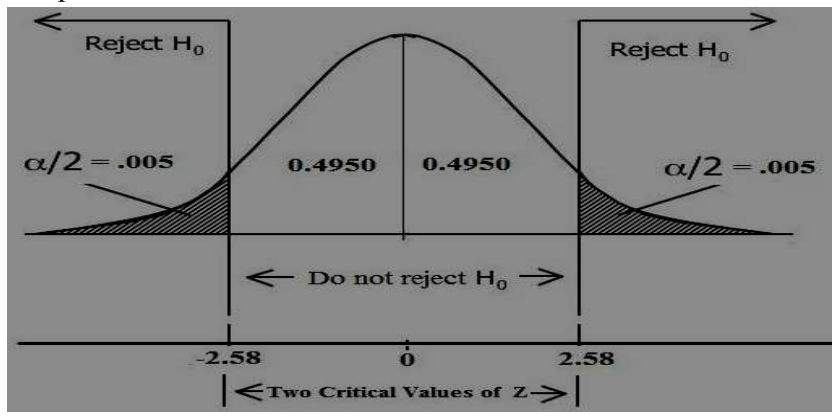

Figure 7. The rejection and nonrejection regions for hypothesis testing about $\left(\mu_{1}-\mu_{2}\right)$ for PETS 2006, Data set 7, Camera 3 video sequence.

lay between $T_{S}$ value range, then $H_{0}$ is accepted and pixel $p(x, y)$ is classified as self shadow in all three frames as shown in Fig. 9.

\section{EXPERIMENTAL RESULTS}

We analyzed and evaluated the performance of self shadow detection and elimination algorithm for surveillance video sequence frames of IEEE PETS ${ }^{1}$ (Performance Evaluation of Tracking and Surveillance) 2001, 2004, 2006, and 2009 data sets. System has been tested using several sequences of PETS data set among which there are different tracking scenario including indoor and outdoor environments, varied number of people. Results shown here are raw results, without any post treatment.

\footnotetext{
${ }^{1}$ Performance data can be found at

"http://homepages.inf.ed.ac.uk/rbf/CAVIAR/"
} 
For each environment, parameters were set once. Results we have selected represent a snapshot of the algorithm results and are typical of the performance throughout the sequences.
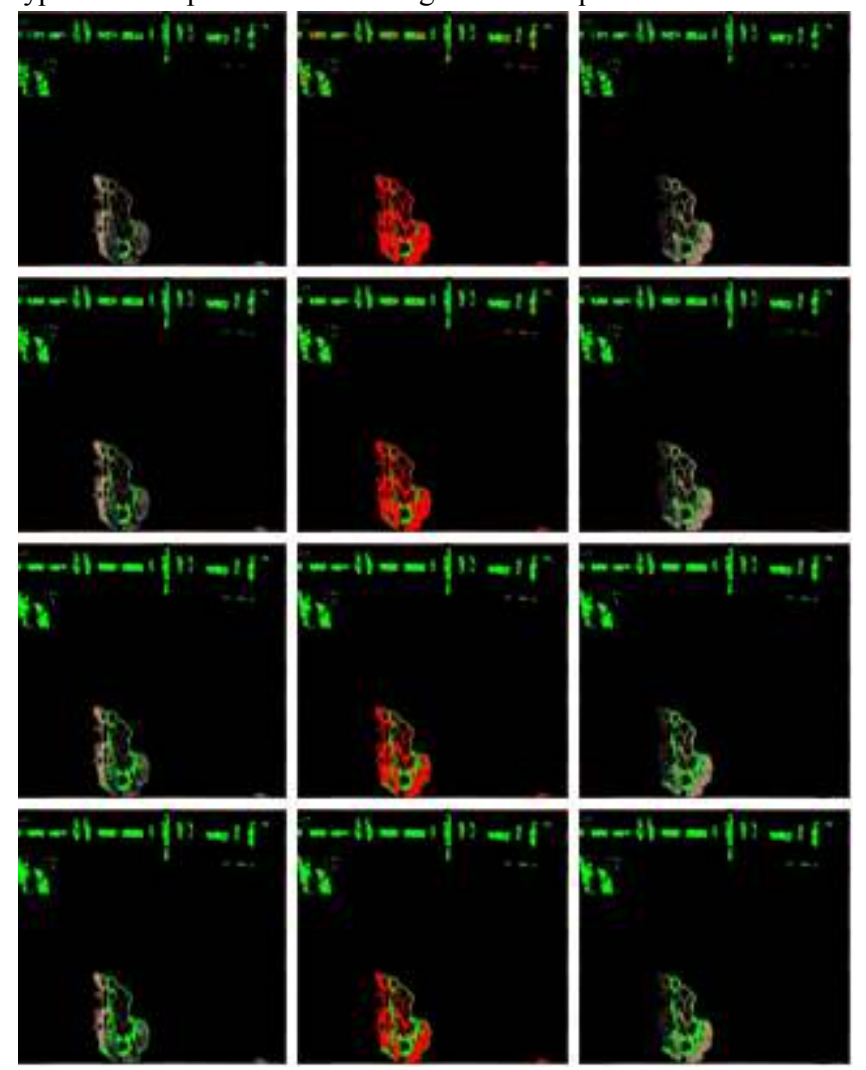

Figure 8. Ts value analysis on the frames $109,112, \& 115$ of the PETs 2006 Data Set 7, Camera 3. Green pixels will be classified as self shadow. First row $=\mathbf{8 0} \%$; Second row $=$ 95\%; Third row $=99 \%$ and Fourth row $=99.9 \%$

In outdoor environments, illumination changes rapidly due to fast changing weather conditions. Figs. 10, 11, 16 and 17 show frames of an outdoor video sequence in which whole image illuminated by direct sun light. This is a particularly challenging situation since the self shadow detection is harder to detect in respect to the other cases. because in an outdoor environment, illumination not only change slowly as daytime progress but may change rapidly due to changing weather conditions as well as passing objects.

There are always variations in the illumination parameters between two frames of the same scene taken even at different times of day. Figs. 12 to 15 show images in indoor environment, corresponding to color video sequences acquired in varying range of fluorescent lighting systems with complex illumination. Because of the multiple light sources on the ceiling and the high reflectivity of the floor, shadows cast on the background by objects have a large variation in intensity. At a given pixel, the shadows go from being fairly light to being fairly deep as a function of the position of the object.

As explained in section 3, a property based shadow description is used for self shadow elimination. Thirty-three challenging PETS video sequences are used to test the proposed system as shown in Table. 3. Each of the sequence contains 2500 to 4000 frames and resolution varied from one sequence to another sequence. Here the comparison of this approach is made based on the visual interpretation, i.e., by looking at processed frames provided by the algorithm. In hypothesis testing type I and type II errors can occur. The type I error occurs when a true $\mathrm{H}_{0}$ is rejected. The value of $\alpha$ represents the probability of committing this type of

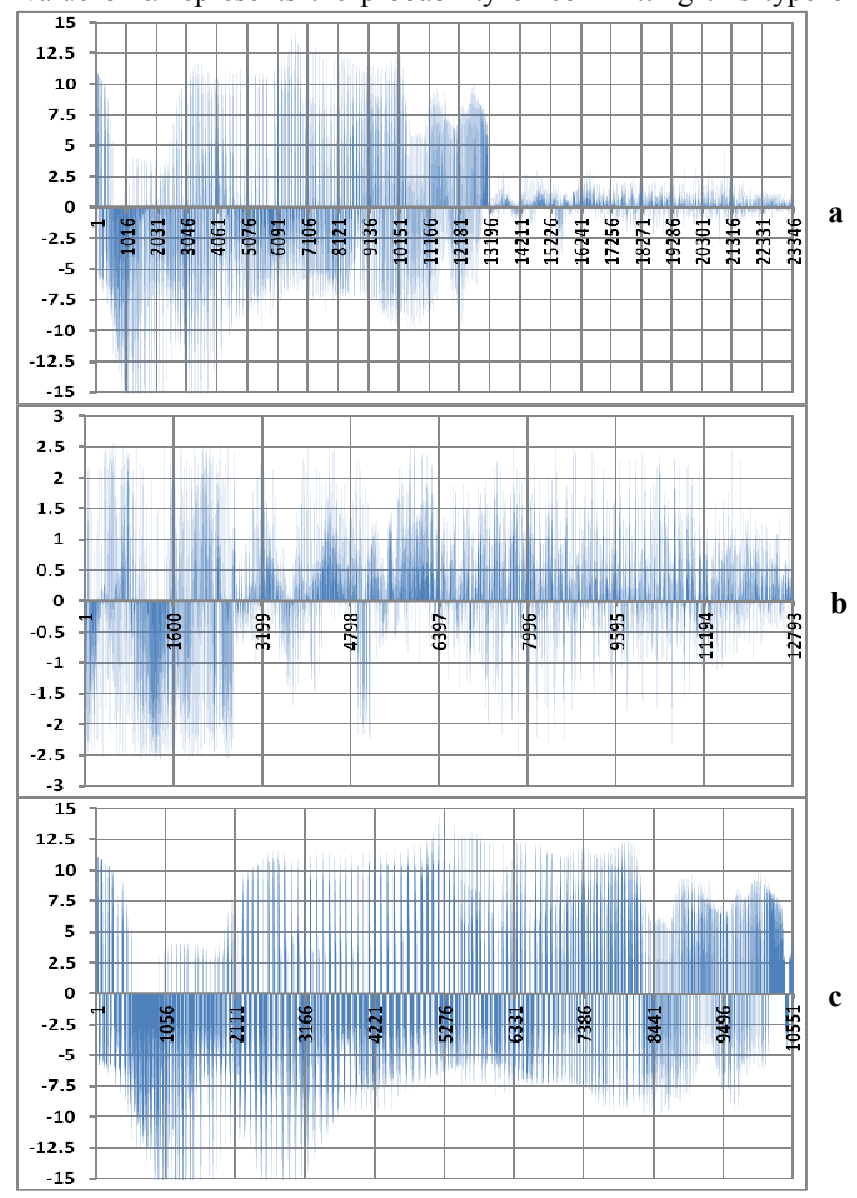

Figure 9. For the frames 109,112 and 115 . The $X$-axis shows number of foreground pixels after motion segmentation [3]. Where, $Y$-axis shows in 9(a) average $Z$ values of foreground pixels, 9(b) and 9(c) shows pixels that are identified as self shadows and motion objects at confidence level $99 \%$ respectively.

error and it represents the significance level of the test. A type II error occurs when a false $\mathrm{H}_{0}$ was not rejected. The value $\beta$ represents the probability of committing a type II error [13].

The $\mathrm{Z}$ test depends on the value assigned to $\alpha$ (Type I error). The rejection region for a hypothesis testing can be on both sides (if the $\mathrm{H}_{1}$ has $\mathrm{a} \neq$ sign), with nonrejection region in the middle as shown in Fig. 7. The size of the rejection region in a statistics problem of a test of hypothesis depends on the value assigned to $\alpha$. We assign a value to $\alpha$ before making the test. Although any value can be assigned to $\alpha$, the commonly used value in this paper for $\alpha$ are $0.01,0.025,0.05$, and 0.10 .

Table 1. Pixels Classification based on Ts value

\begin{tabular}{|c|c|c|c|c|}
\hline $\begin{array}{c}\mathbf{Z} \\
\text { Critical } \\
\text { value }\end{array}$ & $\begin{array}{c}\text { Confidence } \\
\text { Level }\end{array}$ & $\begin{array}{c}\text { Foreground } \\
\text { Pixels after } \\
{[3]}\end{array}$ & $\begin{array}{c}\text { Self } \\
\text { Shadow } \\
\text { pixels }\end{array}$ & $\begin{array}{c}\text { Motion } \\
\text { pixels }\end{array}$ \\
\hline \pm 1.28 & $80 \%$ & 23352 & 10026 & 13326 \\
\hline \pm 1.96 & $95 \%$ & 23352 & 11864 & 11488 \\
\hline \pm 2.58 & $99 \%$ & 23352 & 12794 & 10558 \\
\hline \pm 3.3 & $99.9 \%$ & 23352 & 13721 & 9631 \\
\hline
\end{tabular}


Table 2. Processing time of proposed system to segment motion objects from surveillance video frames.

\begin{tabular}{|c|c|c|c|c|c|c|c|c|}
\hline $\begin{array}{c}\mathrm{Z} \\
\text { Critical } \\
\text { value }\end{array}$ & $\begin{array}{l}\text { Confidence } \\
\text { Level }\end{array}$ & $\begin{array}{l}\text { Processing } \\
\text { time for both } \\
\text { motion } \\
\text { segmentation } \\
\text { \& self } \\
\text { shadow } \\
\text { elimination }\end{array}$ & $\begin{array}{c}\text { Processing } \\
\text { time for } \\
\text { first Spatial } \\
\text { Clustering }\end{array}$ & $\begin{array}{c}\text { Processing } \\
\text { time for } \\
\text { Cast } \\
\text { Shadow } \\
\text { Elimination }\end{array}$ & $\begin{array}{l}\text { Processing } \\
\text { Time for } \\
\text { second } \\
\text { Spatial } \\
\text { Clustering }\end{array}$ & $\begin{array}{l}\text { Total } \\
\text { Time } \\
\text { required } \\
\text { for the } \\
\text { proposed } \\
\text { Scheme }\end{array}$ & $\begin{array}{c}\text { Processing } \\
\text { time for both } \\
\text { motion } \\
\text { segmentation } \\
\text { \& Self } \\
\text { Shadow } \\
\text { elimination }\end{array}$ & $\begin{array}{l}\text { Total } \\
\text { time for } \\
\text { single } \\
\text { frame }\end{array}$ \\
\hline \multicolumn{7}{|c|}{$\begin{array}{c}\text { Average time in milliseconds to process } K,(K+3) \text { and }(K+6) \text { frames. However, we leave } 6 \\
\text { frames while processing. So, in total } 9 \text { frames are processed. }\end{array}$} & \multicolumn{2}{|c|}{$\begin{array}{c}\text { Average time in } \\
\text { milliseconds to process } \\
\text { Single Frame }\end{array}$} \\
\hline \pm 1.28 & $80 \%$ & 351 & 163 & 31 & 70 & 615 & 39 & 68 \\
\hline \pm 1.96 & $95 \%$ & 335 & 171 & 39 & 62 & 607 & 37 & 67 \\
\hline \pm 2.58 & $99 \%$ & 343 & 164 & 39 & 62 & 608 & 38 & 67 \\
\hline \pm 3.3 & $99.9 \%$ & 359 & 171 & 31 & 70 & 631 & 39 & 70 \\
\hline
\end{tabular}

As shown in Fig. 7, a two tailed test has two rejection regions, one in each tail of the distribution curve. The area of each of the two rejection region is $\alpha / 2$ and the total area of both rejection regions is $\alpha$. As shown in Fig. 7, a two tailed test of hypothesis has two critical values that separate the two rejection regions from the nonrejection region. We will reject $\mathrm{H}_{0}$ if the value of $\mathrm{Z}$ falls in either of the two rejection regions. We will not reject $\mathrm{H}_{0}$ if the value $\mathrm{Z}$ lies in the nonrejection regions.

The Fig. 8 shows processed frames by varying $T_{S}$ value to test $\alpha$ and $\beta$ errors. In Fig. 8 green pixels are recognized as self shadows and remaining pixels as motion pixels as shown in Table 1. At $80 \%$ and $95 \%$ confidence interval, some self shadow pixels are classified as foreground pixels. Both self shadow and foreground pixels are classified correctly at $99 \%$ confidence interval. However, at $99.9 \%$ confidence interval some true foreground pixels are misclassified as self shadows. It shows that, two types of errors that occur in tests of hypotheses depend on each other. We cannot lower the values of $\alpha$ and $\beta$ simultaneously for a test of hypothesis for a fixed sample size. Lowering the value of $\alpha$ will raise the value of $\beta$, and lowering the value of $\beta$ will raise the value of $\alpha$ as shown in Fig. 8. However, we can decrease both $\alpha$ and $\beta$ simultaneously by increasing the sample size [14].

In Figs. 10 to 17, first row shows input frames; second row shows motion segmented output frames from [3], which contains self shadows; third row shows frames completely free from self shadows using proposed methodology of this paper; fourth row contains cast shadow eliminated frames using [15].

The proposed scheme aimed at use in surveillance, speed is critical. Table 2 shows, the average time taken to process three (in total 9 frames) temporal differencing frames $\mathrm{K},(\mathrm{K}+3)$ and $(\mathrm{K}+6)$ from PETS 2006 data set 7, camera 3, video sequence for 3500 frame for the original motion detection. This test was performed on a $1.8 \mathrm{GHZ}$ Intel Core 2 Duo processor, where the proposed scheme is implemented using $\mathrm{VC}++$. Input images were $720 \times$ 576 pixels in size and were loaded from disk for the tests.

The proposed algorithms so far to eliminate self shadows $[5,7,8$, $9,10]$ assumes predefined environment and does not work for dynamic environments except for [11]. However, [11] assumes self shadows occurs only on segmented motion object, which is practically impossible as shown in Fig. 2. The proposed property based self shadow elimination algorithm in this paper out performs existing methods on the basis; it works robustly for different dynamic environments and clearly separates self shadows from foreground as shown in Figs. 10 to 17. Moreover, it requires on an average $67 \mathrm{~ms} /$ frame for motion segmentation and shadow elimination (for both self and cast) as shown in Table. 2.

\section{CONCLUSION}

We have presented a novel pixel-based statistical approach, which considers sample pixel from segmented motion objects while calculating $\mathrm{Z}$, which is used consequently, to eliminate self shadows. The proposed shadow removal technique is applied to foreground rather than the entire image so as to save significant processing time. This is important for real time applications such as surveillance systems.

Extensive experiment conducted on different data sets of PETS (to name a few: Outdoor- 10694 frames, Indoor - 65,000 frames) reveals that results are stable and satisfactory. The experimental results showed that the proposed method could detect self shadows in different kinds of scenarios containing strong shadows (occlusion of direct sunlight) and weak shadows (occlusion of indirect light). Using very few parameters, the algorithm clearly captures shadowed background surfaces. Therefore, we conclude that the proposed algorithm works well under various conditions.

\section{REFERENCES:}

[1] Thomas B. Moeslund et al, "A Survey of Advances in Vision Based Human Motion Capture and Analysis", Computer Vision and Image Understanding, October 2006.

[2] Ronald Pope, "Vision Based Human Motion Analysis: An Overview", Computer Vision and Image Understanding. January 2007.

Table 3. Data sets used

\begin{tabular}{|c|c|c|c|}
\hline $\begin{array}{c}\text { PETS } \\
\text { Data set }\end{array}$ & Video Sequence & $\begin{array}{c}\text { No of } \\
\text { Frames }\end{array}$ & Environment \\
\hline 2001 & Camera 1 & 2694 & OUTDOOR \\
\hline 2004 & Shopping Mall & 9000 & INDOOR \\
\hline 2004 & Lab & 7000 & INDOOR \\
\hline 2006 & Camera 3 & 24500 & INDOOR \\
\hline 2006 & Camera 4 & 24500 & INDOOR \\
\hline 2009 & Tracking & 4000 & OUTDOOR \\
\hline 2009 & Tracking & 4000 & OUTDOOR \\
\hline
\end{tabular}


[3] Girisha R and Murali S., "Segmentation of Motion Objects from Surveillance Video Sequences using Temporal Differencing Combined with Multiple Correlation ", Proceedings of the Sixth IEEE Computer Society conference on Advanced Video Signal and Surveillance, Genova, Italy, 02-04 September 2009.

[4] Andrew Woo, Pierre Poulin and Alain Fournier, "A survey of shadow algorithms", IEEE computer graphics \& applications, Volume 10, Issue 6, Nov. 1990 Page(s):13 32 November 1990.

[5] Caixia Jiang and M.O. Ward, "Shadow identification", Proceedings of the IEEE Computer Society Conference on Computer Vision and Pattern Recognition, 15-18 June 1992 Page(s):606 - 612.

[6] J. Stauder, R. Mech and J. Ostermann. "Detection of Moving Cast Shadows for Object Segmentation". IEEE Transactions on Multimedia, 1(1):65-76, March 1999.

[7] Elena Salvador, Andrea Cavallaro and Touradj Ebrahimi, "Shadow identification and classification using color models", Proceedings of the IEEE International Conference on Acoustics, Speech, and Signal Processing, Volume 3, 711 May 2001 Page(s):1545 - 1548

[8] Yinlong Sun, "Self shadow and local illumination of randomly rough surfaces", Proceedings of the 2004 IEEE Computer Society conference on Computer Vision and Pattern Recognition, 2004.

[9] Wang. J.M et.al., "Shadow detection and removal for traffic images", Proceedings of the IEEE international Conference on Networking, Sensing \& Control, 2004.

[10] Takeshi Takai, A Maki and T Matsuyama, "Self shadows and cast shadows in estimating illumination distribution", 4th European Conference on Visual Media Production 2728 Nov. 2007 Page(s): 1 - 10

[11] Li Xu, Feihu Qi and Renjie Jiang, "Shadow removal from a single image", Proceedings of the IEEE international Conference on Intelligent Systems Design and applications, 2006.

[12] Douglas C. Montgomery and Geroge C. Runger, "Applied Statistics and probability for engineers", Third edition, John wiley \& Sons, 2003.Stephen B and Ruth B, "Elements of Statistics II: Inferential Statistics", Schaum's Outlines, Tata McGraw-Hill Edition, 2005.

[13] Stephen B and Ruth B, "Elements of Statistics II: Inferential Statistics", Schaum's Outlines, Tata McGraw-Hill Edition, 2005.

[14] Prem S.Mann, "Introductory Statistics", Fifth edition, Wiley India Edition, 2007.

[15] Girisha.R and Murali. S, "Adaptive Cast Shadow Elimination Algorithm Using Standard Scores for Video Surveillance Applications", Proceedings of the Second International Conference on Signal \& Image processing, Mysore, India, pages 497-506, August,12-14 2009.

\section{ACKNOWLEDGMENT}

Authors would like to thank DRDO (Aeronautical Research \& Development Board). This publication is based on the project sponsored by DRDO file number DARO/08/2021515/M/I

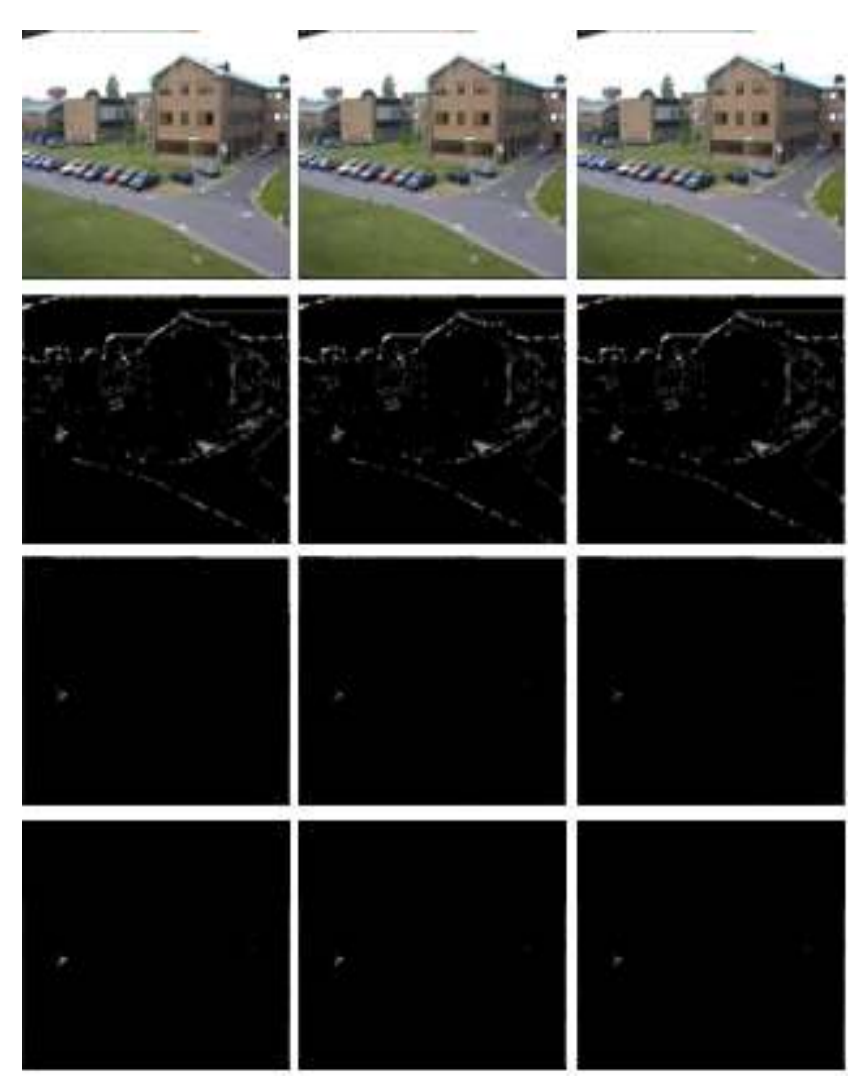

Figure. 10 Frames of PETS data set 2001.
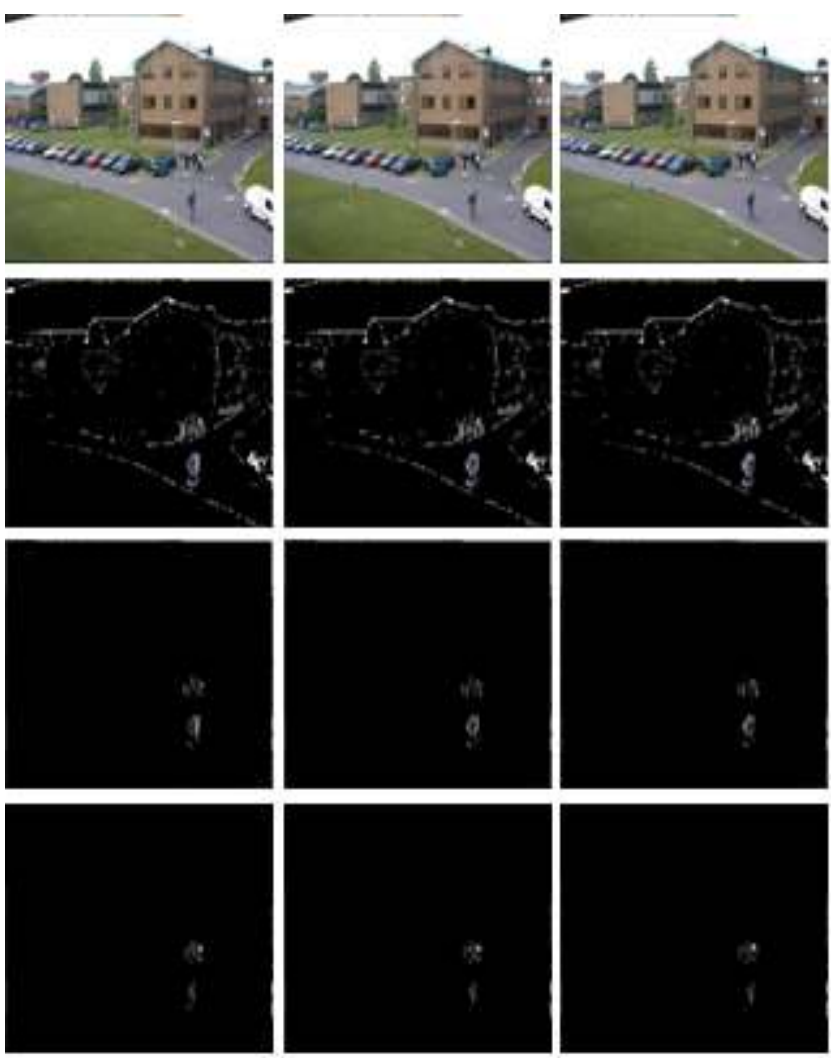

Figure. 11 Frames of PETS data set 2001. 


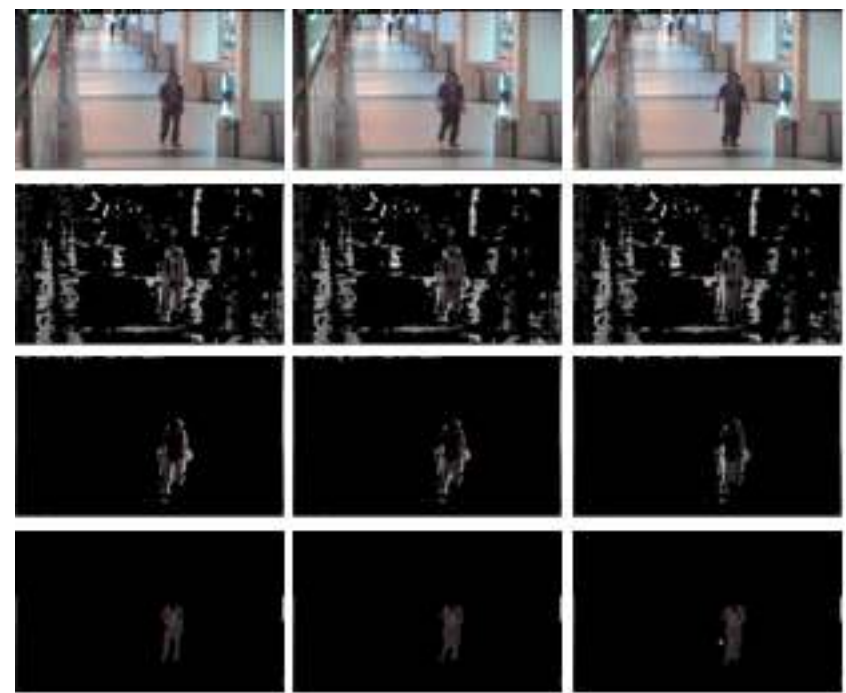

Figure. 12 Frames of PETS data set 2004.
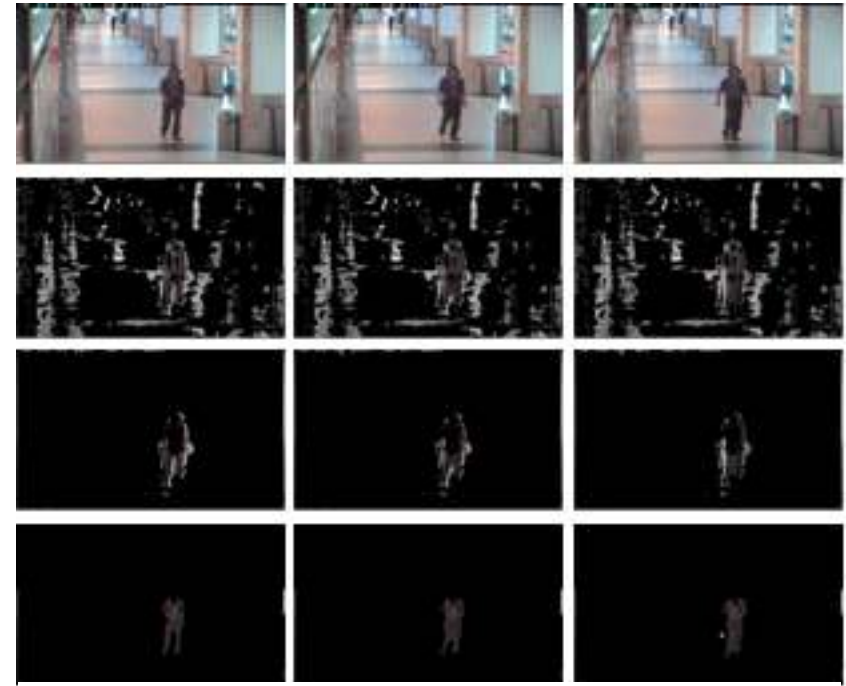

Figure. 13 Frames of PETS data set 2004.

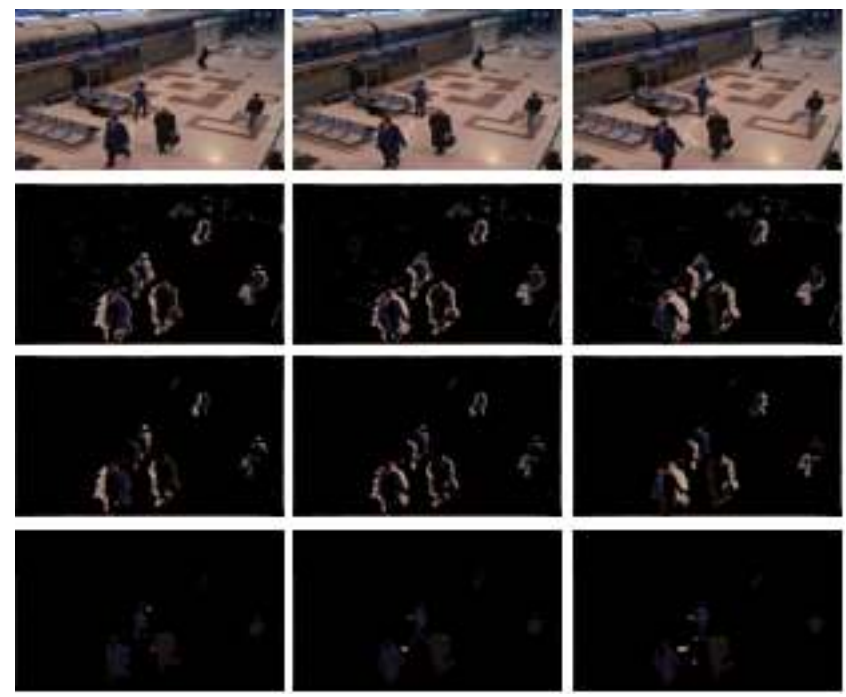

Figure. 14 Frames of PETS data set 2006.

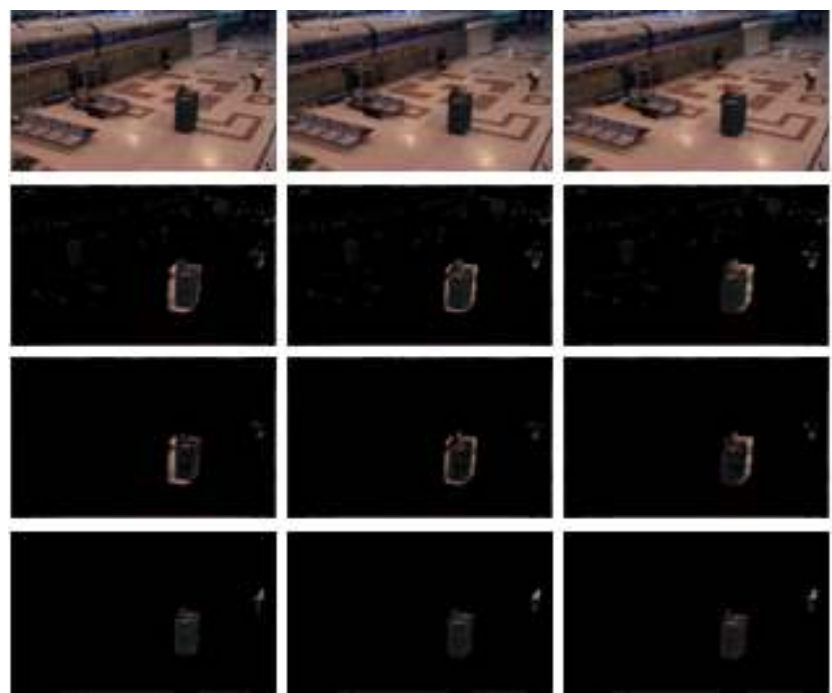

Figure. 15 Frames of PETS data set 2006.

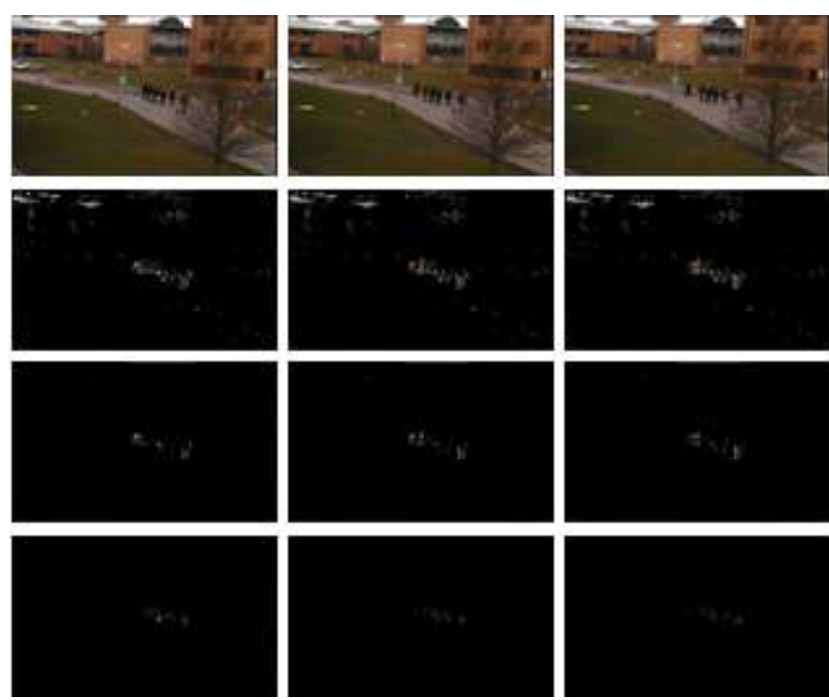

Figure. 16 Frames of PETS data set 2009.

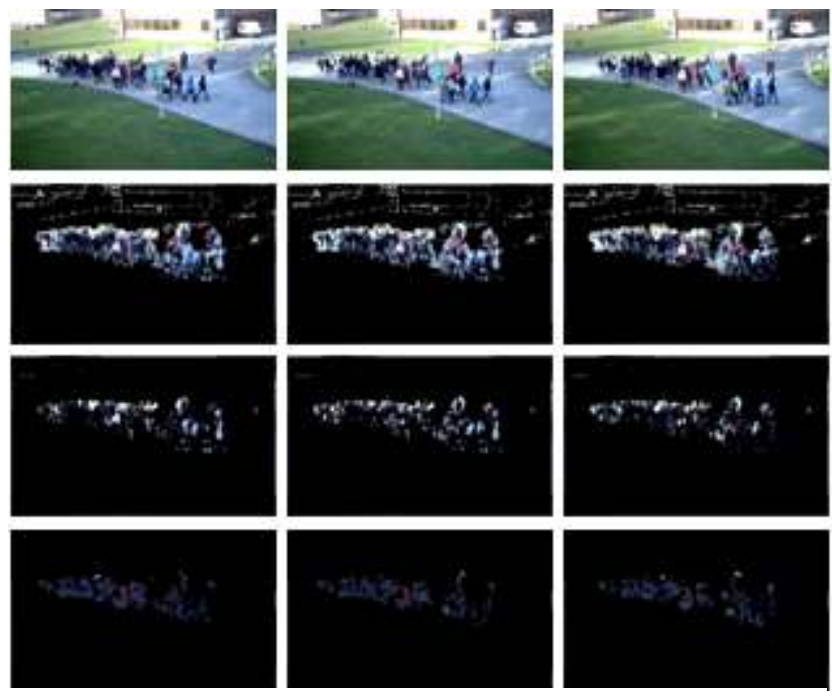

Figure. 17 Frames of PETS data set 2009. 